2. Luks A, Robertson H, Swenson ER. An ultracyclist with pulmonary edema during the bicycle race across America. Med Sci Sports Exerc. In press.

3. Ginsberg JS, Wells PS, Kearon C, et al. Sensitivity and specificity of a rapid whole-blood assay for D-dimer in the diagnosis of pulmonary embolism. Ann Intern Med. 1998; 129(12):1006-1011.

4. Schreijer AJ, Cannegieter SC, Meijers JC, Middeldorp S, Buller HR, Rosendaal FR. Activation of coagulation system during air travel: a crossover study. Lancet. 2006; 367(9513):832-838.

5. De Monye W, Sanson BJ, Mac Gillavry MR, et al. Embolus location affects the sensitivity of a rapid quantitative D-dimer assay in the diagnosis of pulmonary embolism. Am J Respir Crit Care Med. 2002;165(3):345-348.

\section{Reply to High-Altitude Pulmonary Edema Getting Worse After Descent? An Unlikely Event}

\section{To the Editor:}

We appreciate the comments of Luks and Swenson but disagree with their alternative explanations.

With regard to their question of underlying pulmonary hypertension as the cause of respiratory distress, our patient had only trace tricuspid regurgitant jet, and, therefore, no estimation of the pulmonary artery systolic pressure could be made. Although it is true that underlying pulmonary hypertension may lead to a propensity for hypoxia and dyspnea, the patient had no known risk factors for pulmonary hypertension nor other echo findings that would have suggested a cardiac etiology (eg, atrial septal defect) or indirect evidence (eg, right ventricular dilation or dysfunction) of pulmonary hypertension in an otherwise healthy young male.

In terms of the risk for pulmonary embolus, we disagree that the patient had more than a low clinical probability of having a pulmonary embolus. He had a score of 1.5 using the widely accepted Wells clinical decision rule $^{1}$ (heart rate $>100 \mathrm{bpm}$ ), which suggests a low risk of having a pulmonary embolism. In this setting of a low clinical probability along with a negative D-dimer, there is up to a $99.6 \%$ negative predictive value in ruling out a pulmonary embolism in patients presenting to the emergency department, ${ }^{2}$ thus making further imaging unnecessary.

Furthermore, it is unclear if the results of the article involving coagulation factors after air travel ${ }^{3}$ are applicable to high-altitude mountaineering. That study describes subjects who are immobile for 8 hours while in flight. Although both mountaineering and air travel involve hypobaric hypoxia, mountaineering involves vigorous activity, which has an unknown effect on the levels of coagulation factors.
Finally, the patient's clinical presentation and course is truly inconsistent with a diagnosis of pulmonary embolus. A pulmonary embolus significant enough to present with severe hypoxia and diffuse pulmonary infiltrates would not likely be subsegmental or resolve completely over 24 hours.

We believe our patient's clinical diagnosis was most consistent with a late presentation of high-altitude pulmonary edema.

Jared Strote, MD, FACEP Jordan Prutkin, MD

University of Washington Seattle, WA, USA

\section{References}

1. Wells PS, Anderson DR, Rodger M, et al. Derivation of a simple clinical model to categorize patients' probability of pulmonary embolism: increasing the model's utility with the SimpliRED D-dimer. Thomb Haemost. 2000;83:416420.

2. Dunn KL, Wolf JP, Dorfman DM, Fitzpatrick P, Baker JL, Goldhaber SZ. Normal D-dimer levels in emergency department patients suspected of acute pulmonary embolism. J Am Coll Cardiol. 2002;40:1475-1478.

3. Schreijjer AJM, Cannegieter SC, Meijers JCM, Middeldorp S, Büller HR, Rosendaal FR. Activation of coagulation system during air travel: a crossover study. Lancet. 2006;367: 832-838.

\section{Prevention of Acute Mountain Sickness in Nepali Porters: A Controlled Trial}

\section{To the Editor:}

We were delighted to read one of the few research articles on Nepali porters in the Mount Everest region. ${ }^{1}$ Being from this region, we were particularly interested in reviewing the article meticulously, learning from its findings, and applying the information to our medical practice. While studying the article, we came across some points that we hope the authors will clarify.

The first question relates to the methods and the exclusion criteria for enrollment into the study, which were acute mountain sickness (AMS), diabetes mellitus, glaucoma, epilepsy, or sulphonamide allergy. How were these conditions considered? From history, any medical documentation, drugs the porters had taken, or investigations done at the research site? It appears to us that most Nepalese do not know about these conditions.

The second point is the incidence of AMS found: 
$11.9 \%$; this is very low in comparison with previous studies. ${ }^{2}$ Also, AMS was more common in the people taking acetazolamide than in the placebo group. How can this study, therefore, support that acetazolamide prevents AMS?

The third point for consideration is the dropout rate, which the authors opine was related to the porters' possible cultural aversion to taking the drugs. Did the authors find any taboo that discouraged the porters from taking the drugs?

Fourth, the study concludes that there was poor compliance with acetazolamide and that its recommendation to Nepali porters was, therefore, impractical. At the same time, however, the authors suggest that nonpharmacological measures, such as adequate rest days and slow ascent rates, would be good recommendations. In our experience, and as the authors mentioned, these porters are there for employment and must wait for the tourists, thus limiting their options regarding rest days and ascent rate. We therefore believe this recommendation is even less practical.

The final point is that better education has resulted in prompt action being taken after the development of symptoms of AMS and high-altitude cerebral edema in trekkers, ${ }^{3}$ and research shows that large populations of at-risk, high-altitude travelers are relatively naive to the dangers of altitude sickness. ${ }^{4}$ Thus, we believe that better counseling about the dangers of AMS and the importance of taking prophylactic drugs is crucial even in Nepali porters. Were the education and counseling in this study adequate such that the porters fully understood the hazards of AMS and the usefulness of drugs?

\section{Laxmi Vilas Ghimire, MBBS \\ Tribhuvan University Teaching Hospital Kathmandu, Nepal \\ Matiram Pun, MBBS \\ Institute of Medicine Kathmandu, Nepal}

\section{References}

1. Hillenbrand P, Pahari AK, Soon Y, et al. Prevention of acute mountain sickness by acetazolamide in Nepali porters: a double-blind controlled trial. Wilderness Environ Med. 2006;17:87-93.

2. Hackett PH, Roach RC. High-altitude illness. $N$ Engl $J$ Med. 2001;345:107-114.

3. Vardy J, Vardy J, Judge K. Can knowledge protect against acute mountain sickness? J Public Health (Oxf). 2005; 27(4):366-370.

4. Glazer JL, Edgar C, Siegel MS. Awareness of altitude sick- ness among a sample of trekkers in Nepal. Wilderness Environ Med. 2005;16(3):132-138.

\section{Reply to Prevention of Acute Mountain Sickness in Nepali Porters: A Controlled Trial}

\section{To the Editor:}

Drs Ghimire and Pun have made some important points about problems that continue to put Nepali porters at risk of developing acute mountain sickness (AMS). Our answers to their questions are as follows:

1. Exclusion criteria were established solely by taking a history. This can be difficult in any population. Many porters were unaware of the medical conditions mentioned, with the exception of AMS, with which most were familiar. The Nepali doctors, using colloquial language, asked specifically about each exclusion criterion. For example, diabetes was "chini rog," meaning "sugar disease."

2. In the discussion we have touched on the reasons for a low incidence of AMS in these porters. It is also possible that trekking porters are a self-selected group from which AMS sufferers have to some extent been excluded over the years. Because there was such a high dropout rate, the power of the study was so weak that no conclusion could be drawn about the efficacy of acetazolamide.

3. We did not find any evidence of a taboo regarding medication, but as you are probably aware, rural $\mathrm{Ne}$ palis are generally reluctant to take medication, often preferring to trust local healers (shamans). The porters were apparently fit and healthy and sometimes "macho," so we suspect that there might have been some reluctance to take medication despite their initial agreement. Some were persuaded by their sirdars to stop their trial medication. However, we were unable to question many porters who dropped out of the study, so we cannot give a precise answer.

4. We agree that porters' options are limited by the demands of trekking schedules, but this has to change. Sick porters should not be forced to continue trekking and then be abandoned to descend alone. We hope that the results of our study will increase local awareness, thereby supporting the efforts of such organizations as the International Porters Protection Group (IPPG) and Porters' Progress, who are trying to improve the education and working conditions of Nepali porters. The Mountain Medicine Society of Nepal could play an important role in advising and working 